\title{
Numerical Simulation of Maneuvering Aircraft by Aerodynamic, Flight Mechanics and Structural Mechanics Coupling
}

\author{
Andreas Schütte, Gunnar Einarsson, Axel Raichle, Britta Schöning \\ German Aerospace Center (DLR), Institute of Aerodynamics and Flow Technology \\ Lilienthlplatz 7,38108 Braunschweig, Germany \\ Email: Andreas.Schuette@dlr.de \\ Matthias Orlt \\ German Aerospace Center (DLR), Institute of Aerodynamics and Flow Technology \\ Bunsenstrasse 10, 37073 Göttingen, Germany \\ Jens Neumann, Jürgen Arnold \\ German Aerospace Center (DLR), Institute of Aeroelasticity \\ Bunsenstrasse 10, 37073 Göttingen, Germany \\ Wulf Mönnich \\ German Aerospace Center (DLR), Institute of Flight Systems \\ Lilienthlplatz 7,38108 Braunschweig, Germany \\ and \\ Tomas Forkert \\ German Aerospace Center (DLR), Simulation and Software Technology (SISTEC) \\ Linder Höhe, 51147 Köln, Germany
}

Selected results of the DLR Project SikMa-"Simulation of Complex Maneuvers" are presented. The objective of the SikMa Project is to develop and validate a numerical tool to simulate the unsteady aerodynamics of a free flying aeroelastic combat aircraft, by use of coupled aerodynamic, flight mechanics and aeroelastic computations. To achieve this objective, the unstructured, time-accurate flow-solver TAU is coupled with a computational module solving the flight mechanics equations of motion and a structural mechanics code determining the structural deformations. The numerical results are validated by experimental data. For this purpose several specific wind tunnel experiments with different wind tunnel models are carried out.

\section{Nomenclature}

$\Theta$

$l_{\mu}$

$c_{p}=\left(p-p_{\infty}\right) / q_{\infty}$
$=$ Incidence angle, pitch angle at $\Phi=0^{\circ}$

$=$ Angle of attack

$=$ Angle of attack amplitude

$=$ Roll angle

$=$ Initial roll angle

$=$ Yaw angle

$=$ Flap deflection angle

$=$ Reference area

$=$ Chord length of the model

$=$ Aerodynamic mean chord

$=$ Pressure coefficient 


$\begin{array}{ll}q_{\infty}=\rho_{\infty} / 2 \cdot V_{\infty}^{2} & =\text { Dynamic pressure coefficient } \\ c_{M}=M /\left(q_{\infty} \cdot F \cdot l_{\mu}\right) & =\text { Pitching moment coefficient } \\ c_{L}=L /\left(q_{\infty} \cdot F\right) & =\text { Lift coefficient } \\ c_{l}=l /\left(q_{\infty} \cdot F \cdot l_{\mu}\right) & =\text { Roll moment coefficient } \\ M_{x} & =\text { Rolling moment } \\ M_{y} & =\text { Pitching moment } \\ M & =\text { Mach number } \\ V_{\infty} & =\text { On flow velocity } \\ R e=V_{\infty} \cdot l_{i} / v & =\text { Reynolds number } \\ v & =\text { Kinematic viscosity } \\ f & =\text { Frequency } \\ t & =\text { Time } \\ d y & =\text { Deformation increment in y-direction } \\ d z & =\text { Deformation increment in z-direction }\end{array}$

\section{Introduction}

THE improvement of maneuverability and agility is a substantial requirement of modern fighter aircraft. Currently, roll-rates of $200 \%$ and more can be achieved, especially if the design of the aircraft is inherently unstable. Most of today's and probably future manned or unmanned fighter aircraft will be delta-wingconfigurations. Already at medium angles of attack the flow field of such configurations is dominated by vortices developed by flow separation at the wings and the fuselage. The delay in time of vortex position and condition to the on-flow conditions of the maneuvering aircraft can lead to significant phase shifts in the distribution of loads. In such a case, reliable results for the analysis of the flight properties can only be achieved by a combined non-linear integration of the unsteady aerodynamics, the actual flight motion, and the elastic deformation of the aircraft structure.

The main objective of this paper is to focus on the necessity for developing a multidisciplinary engineering tool for predicting the unsteady critical states of complex maneuvering aircraft. Such a simulation environment has to bring together aerodynamics, aeroelasticity and flight mechanics in a time-accurate simulation tool. In order to deliver such a tool, the DLR Project SikMa-"Simulation of Complex Maneuvers" has been initiated to combine these three disciplines into one simulation environment. ${ }^{1,2}$

For validating the numerical simulations several wind tunnel experiments in both the low speed and transonic flow regime have been done within the SikMa project.

\section{Numerical Approach}

\section{A. CFD Solver TAU}

The behavior of the fluid flow affecting the object of interest is simulated with the TAU-Code, a CFD tool developed by the DLR Institute of Aerodynamics and Flow Technology., ${ }^{3,420}$ The TAU-Code solves the compressible, three-dimensional, time-accurate Reynolds-Averaged Navier-Stokes equations using a finite volume formulation. The Code is based on a hybrid unstructured-grid approach, which makes use of the advantages that prismatic grids offer in the resolution of viscous shear layers near walls, and the flexibility in grid generation offered by unstructured grids. The grids used for simulations in this paper were created with the hybrid grid generator Centaur, developed by Centaur Soft. ${ }^{5}$ A dual-grid approach is used in order to make the flow solver independent from the cell types used in the initial grid. The unstructured grid approach is chosen due to its flexibility in creating grids for complex configurations, e.g. a full-configured fighter aircraft with control surfaces and armament. The TAU-Code consists of several different modules, among which are:

- The Preprocessor module, which uses the information from the initial grid to create a dual-grid and the coarser grids for multi-grid.

- The Solver module, which performs the flow calculations on the dual-grid.

- The Adaptation module, which refines and de-refines the grid in order to capture flow phenomena like vortex structures and shear layers near viscous boundaries, among others.

- The Deformation module, which propagates the deformation of surface-coordinates to the surrounding grid. 
- The motion module, which is used to define the motion of the aircraft model and the relative motion of the control devices.

The main elements of the TAU-Code relevant for the SikMa Project, in this case the Solver, Preprocessor, Adaptation, Deformation and Chimera, have all been designed to work efficiently on massively parallel Linux clusters.

The Solver module contains several upwind schemes, as well as a central scheme with artificial dissipation, which are used for the spatial discretization. For simulations of turbulent flows, the one-equation Spalart-Allmaras and several two-equation turbulence models are implemented. For steady computations either an explicit RungeKutta type time-stepping or an implicit LU-SSOR-scheme ${ }^{6}$ are used in combination with the multi-grid technique. For time-accurate simulations an implicit dual-time stepping approach is used

The TAU-Code can handle simulations containing multiple bodies in relative motion with one another, e.g. motion of control surfaces with respect to the aircraft, by use of a hierarchical motion-node structure, and the overlapping-grid chimera technique. The motion of each body can either be calculated internally by the TAU-Code, or supplied by an external program through a Python implemented external interface.

\section{TAU-Code Module: Deformation}

The Deformation module accepts deformed surface-coordinates either as absolute positions, or as relative displacements from the previous surface grid. User defined, rigid body motions of the surface coordinates can also be used to specify the grid deformation. The input used for the cases presented in this paper is a deformed surfacegrid created by an external program. A deformed surface-grid, containing points with new absolute positions, is written to a file which the Deformation module reads. The deformation of the surface grid is propagated through the primary grid, and a new primary grid is created. The Preprocessor module uses this new primary grid to create the dual fine and coarse grids required by the Solver module.

\section{TAU-Code Module: Adaptation}

The Adaptation module refines and de-refines the primary grid using sensors and indicators that are based on flow-solution variables contained in the latest flow field solution from the Solver module. One of the sensors that have commonly been used in various flow simulations is the total pressure value; however, recently new sensors have been implemented specifically in order to address vortex-dominated flow simulations. These new sensors are the $\lambda_{2}$ criteria and kinematic vortex-number, $\mathrm{N}_{\mathrm{k}}$ criteria, which take into account the rotation and rate-of-strain of the velocity gradient, and the normalized helicity $\mathrm{H}_{n}$, which takes into account the angle between the translational and rotational velocity vectors. The new sensors are currently being evaluated as to their effectiveness in capturing the relevant flow phenomena in a vortex-dominated flow field.

\section{TAU-Code Module: Motion}

The Motion module is not a stand-alone executable but a library of functions that handle the rigid-body translational and rotational transformation matrix calculations for the TAU-Code. The module is built to take advantage of naturally occurring hierarchical motion structures, where for example flaps and slats inherit the motion of the wing to which they are attached. Several modes of motion description are allowed, of which the most common are the following:

- $\quad$ periodic - which allows the user to enter a reduced frequency (usually obtained from experimental data), and describe the motion using a combination of Fourier and polynomial series. For periodic motions the user has to specify the number of time-steps per period, such that the Motion module can calculate the maximum time-step allowed, based on the specified reduced frequency.

- $\quad$ rigid - which allows the user to specify a physical time-step size while using the same type of motion description as for the periodic motion. For periodic motions the user has to calculate the appropriate timestep based on the desired number of time-steps per period. For non-periodic motions the user can select a time-step which can sufficiently resolve the prescribed motion.

- rotate - which allows the user to specify a constant rotation around a given axis using a reduced frequency as input parameter. The user has to specify the number of time-steps per period, such that the Motion module can calculate the maximum time-step allowed, based on the specified reduced frequency.

- external - which allows the user to create motion parameters (angles, rates, translation, displacement) in an external program and send those to the TAU-Code through a Python interface to the Motion module. 
The rotation of a body can be described around either the body-fixed coordinate axis (as defined by the DIN 9300 standard), or around a vector defined in space (a so-called hinge-line vector). The translation of a body is specified in the body-fixed reference frame of the parent-node in the motion hierarchy (the inertial reference frame being the parent-node for the entire simulation).

The Motion module uses the given input to create the transformation matrices required to determine the current position of the surface-grids relative to the inertial system, and the relative position of one grid with respect to another for multi-body simulations

\section{TAU-Code Extension: Chimera Technique}

The chimera technique provides the capability to perform calculations with systems of overlapping grids. By allowing large relative body movement without the need for local remeshing or grid deformation, the technique is invaluable for the simulation of maneuvering combat aircraft, where large-amplitude control surface deflections and/or store release are a standard part of the simulation. An example is shown in Fig. 1 for the X-31 aircraft configuration. The current implementation can handle multi-body simulations where the overlapping grid boundaries have been predefined; a version that allows 'automatic-hole-cutting' is currently under development. The chimera search algorithm, which is based on a state-of-the-art alternating digital tree (ADT), is available for both sequential and massively parallel architectures. A more detailed description of the chimera approach is given by Madrane et al.

\section{B. Flight Mechanics}

For the numerical simulation of the flight mechanics, the simulation environment SIMULA developed at the DLR Institute of Flight Systems is used. ${ }^{8}$ SIMULA provides the three basic functionalities necessary for flight simulation and flight control purposes: trimming, i.e. the determination of the initial state and control values, linearization and stability analysis, and simulation, i.e. the numerical integration of the equations of motion.

Single and multi-body flight mechanics models, ranging from 1 to 6 degrees of freedom, are made available to the simulation by SIMULA. The amount of data that is exchanged between SIMULA and TAU is of a scale that can be easily communicated directly through a TCP/IP socket connection, which is offered by the simulation environment TENT.

\section{Structural Dynamics}

For the coupling of the aerodynamic and structural dynamic simulations and flight mechanics in the time domain, two different and independent approaches have been implemented in order to better evaluate the more appropriate scheme for a given simulation complexity. Both approaches are based on a loose coupling scheme and use different software for the spatial coupling, structural dynamics and flight mechanics simulation. The quality of the coupling is considered by equilibrium verification of loads, energies and work at each physical time step.

For the numerical verification of the coupled procedures, validated FE-Models of the generic delta-wingconfiguration which comprises the flexible delta-wing mounted on the flexible wind tunnel support are available. ${ }^{9,10}$ These models have been developed based on results of both ground vibration and static deformation tests.

\section{Modal Approach}

The approach described here is characterized by the use of the multibody system SIMPACK ${ }^{11}$ to account for the elastic structure as well as the flight mechanics and its loose coupling to the computational fluid dynamics software TAU. It is called modal approach since the structural elasticity is introduced from a modal solution of the discrete finite element model, thus receiving a linearly approximated and reduced elastic model which is based on a small number of modal degrees of freedom only. A reasonable number of structural modes has to be chosen to represent the appropriate dynamic behavior. The data exchanged between the multibody system (MBS) and CFD codes is spatially interpolated with the mesh coupling software $\mathrm{MpCCI}^{12}$ and transferred through a TCP/IP internet socket.

The features of the modal approach comprising the topics of temporal and spatial coupling, structural and flight mechanical models are the following:

- Loose coupling for constant communication interval with CFD as master and MBS as slave process; the underlying temporal coupling scheme is the "Conventional Serial Staggered" (CSS) algorithm. ${ }^{13}$

- MBS time integration method is an implicit BDF2 algorithm.

- Use of conservative and non-conservative, element based interpolation algorithms from the MpCCI coupling library to map the aerodynamic forces and the deformed mesh coordinates, respectively. 
- Description of structural elasticity with a reduced, modal approximation computed from the FE-Model.

- Consideration of all translatory and rotatory degrees of freedom in terms of flight mechanics from the MBS functionality.

The provision of the aerodynamic loads in TAU initiates the coupled computation and acts as master process of the co-simulation (loose coupling). SIMPACK delivers the elastic deformations of the coupling surface as slave process. The deformations are interpolated by $\mathrm{MpCCI}$, and then propagated by the TAU deformation tool into a deformed CFD mesh which is pre-processed for the CFD solver. ${ }^{14}$

\section{Discrete Approach}

The second aeroelastic method is the so called discrete approach. The underlying spatial coupling scheme is conservative with regards to the forces, moments and the work performed on both the aerodynamic and structure dynamic side. Furthermore, it is verified that no dissipation or accumulation of net energy occurs. This means that at each time-step the sum of kinetic and potential energy of the structure mechanical model and the performed work of the forces on the aerodynamic surface and their corresponding deformations are in equilibrium.

The main characteristics of this fluid structure interaction in the time domain are as follows:

- loose coupling of computational fluid dynamics (CFD) and computational structure dynamics (CSD) through file input/output,

- time coupling scheme based on adjusted "Conventional Serial Staggered" (CSS) ${ }^{14}$ algorithm modified with a predictor-corrector scheme in each time step for the transformed structural forces,

- use of an implicit or explicit Newmark algorithm for the time integration of the CSD equations of motion, ${ }^{16}$

- use of different scattered data interpolation methods with and without compact support radius for coupling in space domain, ${ }^{17}$

- description of the structure mechanic behavior by the reduced, discrete FE-Model of the delta-wing and the support.

Within this approach for the numerical integration of the structure mechanics equations of motion with the Newmark algorithm, reduced system matrices MAA (mass matrix) and KAA (stiffness matrix) from a NASTRAN eigenvalue solution are used and updated in each time-step to represent the position change of the delta-wing during the flight maneuver. The reduced system matrices are static condensed matrices by a NASTRAN A-Set. This A-Set distinguishes between dependent and independent degrees of freedom. It is assumed that no external load is applied at those degrees of freedom which are neglected. Furthermore, only the translatory degrees of freedom that are involved in the spatial coupling algorithm are included inside of the matrices. One advantage of this approach is, that all modes of the reduced finite elements structure model and their dependency of each other are considered in the coupling between the different discretized aerodynamic and structure mechanics models.

The software package for this approach is called COUPLING and developed at the DLR Institute of Aeroelasticity. It consists of different subroutines and is written in the MATLAB language. The tool can also be used independently from MATLAB if it has been compiled with the appropriate MATLAB compiler in the C or $\mathrm{C}++$ language. ${ }^{14}$

\section{Integration Framework}

For providing an applicable engineering tool it is necessary to have an integration framework organizing the communication between the applications, the management of the simulation scenarios, the data transfer and the capability to distribute the simulation on different computational platforms adapted for the different numerical codes.

The integration framework TENT ${ }^{18}$ provides a graphical user interface for controlling and monitoring coupled simulation workflows. The various codes used in the SikMa simulations will be made available in the TENT system, where a simulation workflow can be built by connecting icons representing each code using a graphical workflow editor. Java wrappers containing the basic control functionality for the TAU and SIMULA applications are already integrated in the TENT environment. The wrapper for the CSM-Code as well as the extension of the functionality to handle the coupling between all three disciplines is under development.

While TENT is providing the data transfer, the communication between the applications and the distribution of the applications on different computational platforms, the communication logic for the simulation workflow is contained within a coupling manager script. The coupling manager is a user-extensible script based on a Python and 
Java interface, where functionality to control the flow of the simulation has been implemented. In Fig. 2 the graphical user interface of the pre-processing tool SimBrowser is shown. The SimBrowser provides the capability to setup the model hierarchy, to define the motion of each element (e.g. flaps, rudder...) and delivers the necessary motion- and hierarchy-input files for the flow solver.

\section{EXPERIMENTAL DATA}

For the validation of the numerical simulation software, various wind-tunnel experiments, designed specifically for the SikMa project, are performed. Experimental data, both steady and unsteady, are available for a $65^{\circ}$-swept delta-wing-fuselage-configuration which has been tested in the DNW Transonic-Wind-Tunnel Göttingen (DNWTWG). The model has movable trailing-edge flaps and can be used for both guided and free-to-roll maneuver simulations around its longitudinal axis. The model shown in Fig. 3 has a chord length of $482 \mathrm{~mm}$ and a span of $382 \mathrm{~mm}$. For the verification of the aerodynamic-structure coupling a static and dynamic system identification of the delta-wing and the support within the wind tunnel is done. The system parameters are used to set up the FE-Model for the coupled simulation.

The main experiments for the SikMa project are done in the DNW Low-Speed-Wind-Tunnel Braunschweig (DNW-NWB). ${ }^{21}$ In order to perform these experiments, a wind-tunnel model has been designed and built for the SikMa project. The model, shown in Fig. 4, is based on the X-31 experimental high angle-of-attack aircraft configuration. The model is equipped with remote controlled movable control devices driven by internal servoengines, as seen in Fig. 5. Measurement equipment is installed to determine the aerodynamic forces and moments on the model, as well as span-wise pressure distributions at locations of $60 \%$ and $70 \%$ chord length. The experiments include steady-state measurements using PSP-"Pressure Sensitive Paint", which provide detailed information on the surface pressure distribution for the whole wing. The experiments will culminate with maneuver simulations, where the movement of the aircraft and the control devices will be synchronized. For the maneuver experiments the model will be mounted on the MPM-"Model Positioning Mechanism" of the DNW-NWB. Fig. 6 shows the X-31-RemoteControl-Model mounted on the MPM-System.

\section{RESULTS}

For the verification and validation of the simulation environment, the results of the numerical simulations are compared against data collected from various experimental simulations. For this purpose a validation strategy is defined considering the model configurations and the test and validation scenarios. One of the model configurations is the delta-wing-configuration described in section III. The model is used to show the capability of the TAU-Code to predict the unsteady aerodynamic behavior of configurations with vortex dominated flow fields. The final configuration is the X-31 model. Results for the delta-wing-configuration will be shown for verification of the coupling procedures, after which results for the validation of steady and unsteady simulations of the X-31 configuration will be presented.

\section{A. Verification of the Coupling Procedure}

In Fig. 7 a CFD - flight-mechanics coupled simulation of the delta-wing with trailing edge flaps is depicted. The initial attitudes are $\alpha=17^{\circ}$ and $\Phi_{0}=0^{\circ}$. The trailing-edge flaps are deflected by $\eta= \pm 5^{\circ}$ once the model has been released. The turbulence model used for this simulation is the k- $\omega$-model with the Kok-TNT-rotational correction approach. ${ }^{19}$ Two calculations are done using this configuration. The first calculation is done without taking into account the effects of mechanical friction, while for the second calculation a mechanical friction of $3.5 \mathrm{Nm}$, which is approximately determined from the experimental data, is implemented. The characteristic movement of the model, as well as the roll-moment coefficient, is well predicted by the second calculation. By analyzing the roll-moment coefficient we observe the following:

- An asymmetric surface force-distribution develops due to the asymmetric trailing-edge flap deflection, which in turn leads to a rotational acceleration around the longitudinal axis of the model.

- The maximum roll-moment coefficient is reached after a simulation time of $0.05 \mathrm{~s}$, where the flaps are at $\eta=2.5^{\circ}$ deflection. After this the roll-moment coefficient decreases and reaches a temporary plateau at $\mathrm{t}=$ $0.1 \mathrm{~s}$, at which time the flaps are fully deflected at $\eta=5^{\circ}$. 
- The model reaches a trim-point at $\Phi=31^{\circ}$, where the combined roll-moment is not large enough to overcome the aerodynamic damping due to the asymmetric loads on the wing together with the mechanical friction of the system.

The reason for the movement of the model is graphically explained in Fig. 8. At the start of the simulation the wing is accelerated due to the asymmetric flap deflection, see Fig. 8.1. The vortex on the luff side of the wing is strengthened with the increasing roll angle. The effective sweep angle on the luff side of the wing is decreasing, which in turn increases the normal component of the on-flow vector. This causes a stronger primary vortex on the luff side, which is located closer to the surface, thus leading to a higher local lift on the luff side. On the lee side the opposite effect happens. The wing vortex gets weaker and the distance from the wing surface higher as the roll angle increases, which leads to a lower local lift on the lee side, see Fig. 8.2. This effect causes the wing to decelerate, which in turn leads to the trim-point at $\Phi=31^{\circ}$, Fig. 8.3 and 8.4.

This example shows the capability of CFD - flight-mechanics coupling by means of a delta-wing with trailing edge flaps. The main aerodynamic effects are qualitatively well predicted, but in order to predict the quantitative aerodynamic values obtained experimentally it is necessary to have the same starting conditions and environment parameters that were in effect during the experimental maneuver scenario.

The capability to predict the elastic deformations accurately is demonstrated for the delta-wing-configuration during a guided roll maneuver for both the discrete and modal approach. The delta-wing and the rear-sting support are considered to be elastically deformable within the coupled simulation between the TAU-Code and the structural dynamics tools. For the coupled simulation, the finite element model ${ }^{10}$ takes into account both the delta-wingconfiguration as well as the flexible support, as seen in Fig. 9. The FE-Model is validated based on results of both ground vibration and static deformation tests. The aerodynamic calculations are performed in Euler mode using a coarse mesh topology for the reduction of computation time on the CFD side. This procedure is sufficient to verify the general capabilities of the two developed approaches.

The guided maneuver is a constant rotation at $5 \mathrm{~Hz}$ of the wing body around its own longitudinal axis. The on flow velocity is at a Mach number of 0.5 and the pitch angle, i.e. angle-of-attack for zero roll angle, is $6^{\circ}$. The corresponding history of the model deflection is depicted in Fig. $\mathbf{1 0}$ for the sting support at the trailing-edge position of the delta-wing. It can be seen that the sting is undergoing an elliptical-like motion during the guided maneuver. The green curve in Fig. 10 shows the sting movement from the experiment which is integrated from the measured accelerations. A comparison of the numerical results computed from discrete and modal approach shows a good agreement of the predicted motion at the sting position with the measured data. Due to the elastic deformation, which is rather dominated by the model sting bending than the deflection of the generic delta wing, the effective angle of attack has increased for a given roll angle leading in turn to higher values for the rolling moment coefficient, as presented in Fig. 11.

The time-history results for the rigid and the coupled elastic simulation, performed with the modal approach, for the free-to-roll wind tunnel maneuver are compared in Fig. 12 for roll angle $\Phi$ and rolling moment $M_{x}$, respectively. The on-flow Mach number is at $\mathrm{M}=0.5$, the pitch angle is $9^{\circ}$ and the initial roll angle is at $45^{\circ}$. As described before, the maneuver is initiated by the unsymmetrical load distribution over the wing surface due to the initial roll angle. Furthermore, similar elastic effects occur as already found with the simulation of the guided maneuver. The elastic deformations of the support in lateral y- and vertical z-direction lead to locally increased angles of attack and thus to larger aerodynamic loads resulting in a changed rolling moment around the body-fixed x-axis of the delta-wing, according to Fig. 12 and 13. In contrast to the rigid result, the elastic simulation shows a roll angle with an offset of approximately $2.5^{\circ}$. The reason is found in the pitch- and yaw-like contributions from the elastic deflection of the axis of rotation which is considered for the computation of the aerodynamic loads. Within further RANS calculations comparisons with experimental data will be done.

In Fig. 14 the delta-wing-model is used to show the trimming capability of the CFD - flight-mechanics coupling procedure. The initial conditions are shown in Fig. 15: $\mathrm{M}=0.5, \Theta=17^{\circ}, \Phi=30^{\circ}, \eta_{0}=0^{\circ}$. The trimming algorithm uses a gradient-based approach to determine the direction and magnitude of the flap deflection. The desired trimming condition is a rotational acceleration magnitude of zero around the longitudinal axis of the delta-wing. In Fig. 15 the surface distribution and flow topology of the primary vortices are depicted for the non-trimmed conditions. Due to the asymmetric flow conditions the luff vortex on the left hand side is stronger and closer to the surface of the wing. This causes a local higher lift on the luff side in comparison to the lee side where the vortex is weaker and farther away from the surface. This causes a negative damping rolling moment which has to be compensated by flap deflection. In Fig. 16 the surface distribution and flow topology of the primary vortices are shown for the trimmed 
condition, where the flaps have been deflected by $\eta= \pm 7^{\circ}$. It is seen that the vortex structure is not influenced by the flap deflection. The only significant difference is a higher suction peak over the flap on the lee side of the wing due to the upward deflection of the flap.

\section{B. Validation for the $\mathrm{X}-31$ Configuration}

Having presented the principal capabilities of the coupling procedures by means of the simple delta-wing-model, the next step is to determine the capability to accurately capture the steady and unsteady aerodynamics of the more complex X-31 configuration, by comparing the numerical results with experimental data.

In Fig. 17 the topology of the computational model for the simulation of the X-31 configuration is shown. All control devices are taken into account and two different setups are simulated. The first setup is simulated with a rear sting support. For the second a belly sting support is mounted on the lower side of the model for the unsteady calculations as it is configured in the experiments. In Figure 18 the hybrid mesh topology is depicted. A pre-refined mesh is used for both steady and unsteady simulations. To cover a certain range of pitching and yawing angle the pre-refinement is adapted to the areas where the vortices are assumed during the simulation. The steady state results are simulated with no sting, while the unsteady simulations are simulated with the belly sting support taken into account, as was the case in the experiments. The effect of the support is discussed later on.

Fig. 19 shows the numerically simulated 3D flow field over the X-31 configuration, and gives a good indication of the complexity of the vortex flow topology over the wing and fuselage. Comparisons with experimental data show good agreement regarding the vortex topology. In Fig. 20a an oil flow picture of the X-31 clean-wing from low speed experiments is shown. The angle-of-attack is $\alpha=18^{\circ}$ at a Reynolds number of 1.0Mio. The attachment line of the strake vortex and the main wing vortex as well as the separation line of the main wing vortex near the leadingedge is emphasized. In Fig. 20b the corresponding CFD calculation is depicted. It is seen that the flow topology from the calculation fits quite well with the experiment.

Further calculations were done taking all control devices into account. In Fig. 21a the PSP result at $\alpha=16^{\circ}$ at a Reynolds number of $2.07 \mathrm{Mio}$ is shown. Comparing the pressure distribution from the PSP measurement with the CFD calculation in Fig. 21b it is seen that the main footprints of the vortices as well as the location are captured by the CFD numerics. The calculations were done using the Spalart-Almaras turbulence model with vortex correction. The main difference with respect to the clean-wing configuration is the separated vortex shear layer, which occurs due to the gaps between the leading edge control-devices. This leads to a more complex vortex topology, with three vortices. The first vortex comes from the inner wing, the second and third from the inner and outer flap, respectively. The suction-strength of the vortex at the inner leading edge flap is predicted to be stronger and the outer vortex starting at the kink of the wing is predicted to be weaker than in the experiment.

In Fig. 22 the lift and pitching moment coefficient over the angle of attack is shown. For all calculations at $10^{\circ}$, $12^{\circ}, 14^{\circ}$ and $16^{\circ}$ angle of attack a higher front loading pitching moment is predicted by the numerics, while the overall lift is fairly well predicted by the CFD calculation. The reason for this can be identified by looking at the pressure distribution on the canard and the two stages at $60 \%$ and $70 \%$ cord length in Fig. 23 to 26 . For $12^{\circ}$ angle of attack and higher, the suction peak over the canard is predicted too low. This causes a lower contribution for a rear loading pitching moment of the canard. Regarding the pressure distribution on the wing it is seen that overall pressure distribution is well predicted by CFD. However, the formation of the vortex at the outer leading edge flap occurs at a somewhat higher angle of attack than in the experiment. The difference between experiment and numerical simulation is easily observable in Fig. 28 at $70 \%$ cord length, where no outer vortex is shown by the numerics while in the experiment a high suction vortex peak is measured.

Fig. 27 shows the result of a guided yawing motion maneuver. The initial pitching angle is $10^{\circ}$ and the yaw amplitude is $\Delta \beta=5^{\circ}$. The maneuver is done with a frequency of $1 \mathrm{~Hz}$. In this scenario the model is mounted on the belly sting support described above. The overall aerodynamic behavior is captured by the numerical simulation, although the pitching moment is under-predicted. The gradient of the rolling moment is predicted to be higher than in the experiment and the hysteresis shown in the experiment is predicted to be lower by the calculation. Comparing the initial pitching moment in Fig. 27 at $10^{\circ}$ in comparison to the steady state results in Fig. 22 it is seen that the pitching moment in case of the belly sting is more than double that without sting. The reason for this is that the belly sting is shifting the local angle of attack of the inner wing to higher values. This causes a higher suction and a 
corresponding higher front loading on the wing. Furthermore, the induced angle of attack of the canard is rising, which leads to a higher lift on the canard and higher rear loading pitching moment.

Fig. 28 shows the result of a guided pitching motion maneuver. The initial pitching angle in this case is $10^{\circ}$ with an amplitude of $\Delta \alpha=4^{\circ}$. The maneuver is again done with a frequency of $1 \mathrm{~Hz}$. As in case of yawing motion the lift is predicted higher than in the experiment. The gradient is predicted correctly. Unfortunately the characteristic of the dynamic pitching moment is not given precisely by the numerical simulation. The reason is the same as described in the steady state case before. Fig. 29 and $\mathbf{3 0}$ show the flow topology at the lower and upper points of the dynamic loop. In Fig. 29 the flow is attached over the wing at $\alpha=6^{\circ}$ and even the flow around the canard is attached. Only a small vortex is generated from the strake in front of the inner wing. In Fig. $\mathbf{3 0}$ at $\alpha=14^{\circ}$ the flow topology has changed over all. Over the wing a vortex structure of four vortices starting from the wing strake, at the inner, from the inner and outer leading edge flap, has developed. The inner wing vortex and the vortex from the inner leading edge flap unify at approximately $70 \%$ cord length. Over the canard a strong vortex is generated which is not interacting with the wing vortices. The small strakes at the nose of the fuselage prevent the appearance of rolling instabilities caused by time dependant flow-separations.

Having provided initial validation results showing the capability to predict the overall flow topology around the $\mathrm{X}-31$ configuration, the scenarios are extended to CFD - flight-mechanics coupled simulations. The initial stage is a trimming simulation for the complex X-31 configuration. The results obtained at the end of the trimming simulation are then the initial state for further free-flight maneuver simulations.

Fig. 31 shows the convergence of the pitching-moment, along with the corresponding flap deflection, of a trimming simulation for the X-31 configuration. The initial flight state is as follows: weight of $30 \mathrm{~kg}$, thrust of $50 \mathrm{~N}$, altitude is $10,000 \mathrm{ft}$, Mach Number is 0.3 , angle-of-attack is $5^{\circ}$, pitch-angle is $5^{\circ}$, and trailing-edge flap deflection is $1.72^{\circ}$. The trimming requirements are an angle-of-attack of $5^{\circ}$, with longitudinal acceleration (translational and rotational) of zero. The trim module determines a new flight state dependent on the gradient calculations for the translational velocity components $u$ and $w$, the pitch-angle $\Theta$, and the flap deflection angle. $\eta$. The result of the trimming simulation is the following flight state: Mach number of 0.29 , angle-of-attack of $5^{\circ}$, pitch-angle of $-1.55^{\circ}$, trailing-edge flap deflection of $-0.18^{\circ}$, and a flight-path angle of $-6.55^{\circ}$.

Fig. 32 shows the overlapping chimera-grids around the right trailing-edge flap and the surface pressurecoefficient for the same section at the trimmed flight-state conditions.

In the final application the simulation of a free-to-roll maneuver of a generic aeroelastic model of the X-31 wind tunnel model was performed. The objective of this test case is to show the capability of the implemented fluidstructure-flight mechanic simulation to handle complex configurations. The results for this maneuver are shown in Fig. 33 to Fig. 36. The simulation is a free-to-roll maneuver of the elastic X-31 configuration around the longitudinal axis. The full aircraft configuration with fuselage, wings, and control surfaces, is contained in the structural model. The structure of the X-31 model is generic and has been designed such that the deformations during the flow conditions in the wind tunnel are clearly visible. The finite element model has 273 nodes with 819 discrete translational degrees of freedom and is depicted in Fig. 33. Euler computations have been performed on a mesh with 19.4 million tetrahedrons, 3.44 million nodes and 261792 nodes on the surface. To simulate the free-toroll maneuver around the longitudinal axis a simplified one degree of freedom flight mechanic model was used. This means that the acceleration of the model is dependent only on from the aerodynamic rolling moment $M_{x}$ and the moment of inertia around the $\mathrm{x}$-axis of the finite element model.

The simulated maneuver starts from a roll angle of $45^{\circ}$ and a pitch angle of $15^{\circ}$. A converged, steady-state solution of the aerodynamic forces and moments was obtained for the undeformed mesh. During the maneuver unsteady nonsymmetric loads on the aircraft lead to non-symmetric deformation of the structure. The elastic model is depicted in Fig. 33. Shown are the deflected finite element model and the corresponding transformed aerodynamic forces. In Fig. 34 the comparison of the rolling angle during a real time of 0.5 second between the rigid and the elastic model is shown. It is seen that the rolling angle for the simulation with the elastic model converged quicker to a rolling angle of zero degree. The reason for this is the higher aerodynamic moment $\mathrm{M}_{\mathrm{x}}$ due to the elastic behavior of the finite element model. The deflection of the elastic model due to the aerodynamic forces induces a higher angle of attack and leads to a higher rolling moment. Due to the one degree of freedom flight mechanic model the acceleration of the rolling angle is only dependent on the aerodynamic moment $\mathrm{M}_{\mathrm{x}}$, which is dependent on the elastic deformation. Due to the elastic behavior of the model the rotational acceleration is higher than for the rigid model, which leads to a higher rolling frequency. This in turn leads to a higher aerodynamic damping of the system, which has to effect of bringing the system to a trimmed position within a shorter period of time than is observed with the rigid model. For this simulation the comparison of the corresponding pressure distributions for the rigid and 
the elastic CFD model are shown in Fig. 35. The pressure distributions differ strongly from each other between the rigid and the elastic models. Since the loads on the configuration are asymmetric during the maneuver, the displacements due to the transformed structural loads on the left and right hand side differ from each other. In Fig. 36 the difference of the aerodynamic surface between the rigid and the full elastic model during the maneuver simulation is shown. The maximum displacement at the wing tip is approximately $3.8 \mathrm{~cm}$ for a half span of the model of $50 \mathrm{~cm}$. This simulation has shown the capability of the implemented coupling algorithms and dataexchange logic to simulate a maneuver which relies on high fidelity aerodynamics, finite element modeling and flight mechanics. Within further investigations RANS calculations will be done taking the deformation of the control devices into account.

\section{CONCLUSIONS}

In this paper the activities and recent results of the DLR-Project SikMa were presented. In SikMa a simulation tool should be developed that is capable of simulating a maneuvering elastic aircraft with all its movable control devices. The simulation tool combines time-accurate aerodynamic, aeroelastic and flight-mechanics calculations to achieve this objective. Preliminary verification of the functionality of the simulation tool has been shown by simulating a sharp leading-edge delta-wing during free-to-roll maneuvers due to flap deflections. First perspectives were presented regarding the time-accurate coupling between the TAU-Code, CSM-Code and flight-mechanics. Initial results of the steady and unsteady flow field around the X-31 configuration were presented. The trimming algorithm was demonstrated using the X-31 configuration. Finally, a free-to-roll maneuver was presented using the X-31 configuration in a time-accurate coupling of CFD, CSM and flight-mechanics. Additional simulations will be done to validate the TAU-Code for the X-31 configuration. Therefore all control devices will be taken into account within a maneuver simulation as it was performed in the experiments.

\section{Acknowledgments}

The authors would like to thank Dr. Martin Rein, Dr. Gebhard Höhler, Peter Klemens and Hartmut Haselmeyer for providing the wind-tunnel data and for their engagement managing the experimental simulation tasks at DLR Göttingen within the project SikMa.

\section{References}

1 Schütte, A.; Einarsson, G.; Schöning, B.; Madrane, A.; Mönnich, W., Krüger, W.: Numerical simulation of manoeuvring aircraft by aerodynamic and flight mechanic coupling. RTO AVT-Symposium Paris, 22.-25. April 2002.

2 Schütte, A.; Einarsson, G.; Schöning, B.; Raichle, A.; Mönnich, W.; Neumann, J.; Arnold, J; Heinecke, J.: Numerical simulation of maneuvering combat aircraft. AG-STAB, STAB-Symposium, Nov.2004, Bremen.

3 Galle, M.; Gerhold, T.; Evans, J.: Technical Documentation of the DLR TAU-Code DLR-IB 233-97/A43 1997

4 Gerhold, T.; Galle, M.; Friedrich, O.; Evans, J.: Calculation of Complex Three-Dimensional Configurations employing the DLR TAU-Code. AIAA-97-0167 1997.

5 Centaur Soft: http://www.Centaursoft.com

6 Dwight, R.P.: Time-Accurate Navier-Stokes Calculations with Approximately Factored Implicit Schemes. Proceedings of the ICCFD3 Conference Toronto, Springer, 2004.

Madrane, A.; Raichle, A.; Stürmer, A.: Parallel implementation of a dynamic overset unstructured grid approach. ECCOMAS Conference Jyväskylä Finland, 24.-28. July 2004.

8 Mönnich, W.; Buchholz, J. J.: SIMULA - Ein Programmpaket für die Simulation dynamischer Systeme - Dokumentation und Benutzeranleitung - Version 2 DFVLR Institutsbericht IB 111-91/28, 1991.

9 Hoffmann, D.; Neumann, J.; Sinapius, M.: Strukturmechanische Identifikation von Halterung und Windkanalmodell "AeroSUM". DLR-IB, IB 232-2002-C06, 2002.

10 Neumann, J.: Strukturmechanische und strukturdynamische Finite Element Modelle des Windkanalmodells "AeroSUM" mit Halterung. DLR-IB, IB 232-2003-J01, 2003.

${ }_{11}$ Lugner, P.; Arnold, M.; Vaculin, O. (Eds): Vehicle System Dynamics (Special issue in memory of Professor Willi Kortüm). Vol. 41, No. 5, 2004.

${ }_{12}$ Fraunhofer Institute for Algorithms and Scientific Computation SCAI: MpCCI Mesh based parallel code coupling interface. Specification of MpCCI version 2.0, 2003.

13 Farhat C.; Lesoinne M.: Higher-Order Staggered and Subiteration Free Algorithms for Coupled Dynamic Aeroelasticity Problems. AIAA 98-0516, 1998

14 Arnold, J.; Einarsson, G.; Schütte, A.: Multibody Simulation of an Aeroelastic Delta Wing in Roll Manoeuvres. Proc ICAS 2006, Hamburg, Germany; 2006. 
15 Einarsson, G.; Neumann, J.: Multidisciplinary Simulation of a Generic Delta Wing: Aerodynamic, Flight-Dynamic, and Structure-Mechanic Coupling. ECCOMAS, International Conference on Computational Methods for Coupled Problems in Science and Engineering, Santorini Island, 2005.

16 Schulze, S.: Numerische Integration der aeroelastischen Bewegungsgleichungen eines Flügelprofils. DLR-IB, IB 232-94 J 06, 1994.

17 Beckert, A.; Wendland, H.: Multivariate Interpolation for Fluid-Structure-Interaction Problems using Radial Basis Functions. Aerospace Science and Technology, Art. No. 5125, 2001.

18 Schreiber, A.: The Integrated Simulation Environment TENT. Concurrency and Computation: Practice and Experience, Volume 14, Issue 13-15, S.1553-1568, 2002.

19 Dol, H.S.; Kok, J.C.; Oskam, B.: Turbulence modeling for leading-edge vortex flows. AIAA 2002-0843, Reno, Jan. 2002 .

20 Gerhold, T: Overview of the Hybrid RANS Code TAU, in N. Kroll, J. Fassbender (Eds.) MEGAFLOW - Numerical Flow Simulations for Aircraft, NNFM Vol. 89, Berlin, 2005, pp. 81-92.

21 Rein, M.; Höhler, G.; Schütte, A.; Bergmann, A.; Löser, T.: Ground-Based Simulation of Complex Maneuvers of a Delta-Wing Aircraft. AIAA-2006-3149, 25th AIAA Aerodynamic Measurement Technology and Ground Testing Conference, San Francisco, California, June 5-8, 2006. 


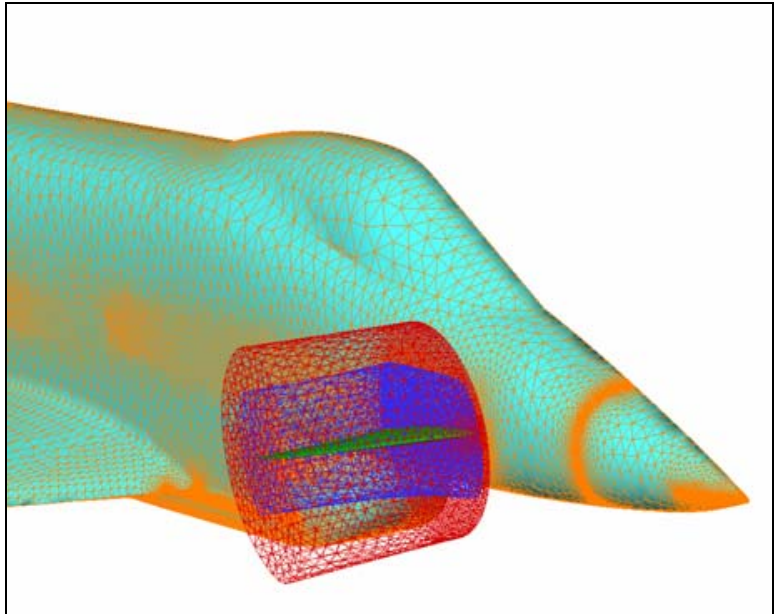

Figure 1: Chimera mesh of the $\mathrm{X}-31$ configuration.

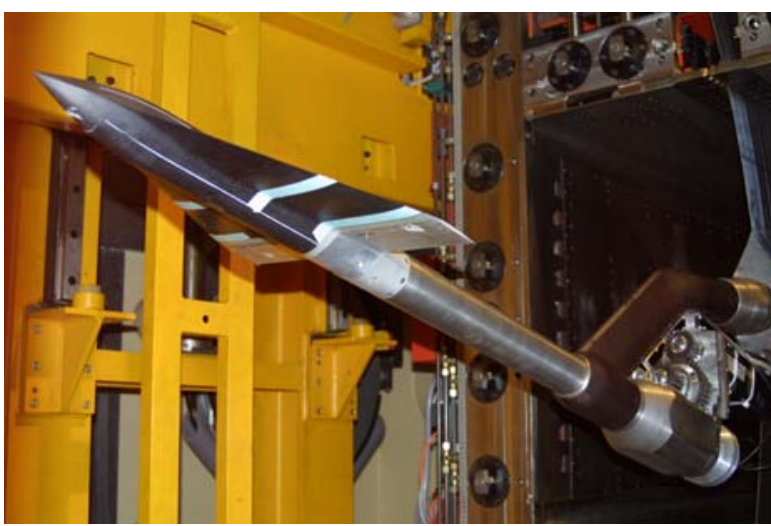

Figure 3: $6^{\circ}$-swept delta-wing-fuselage-modelconfiguration with remote controlled trailing-edge flaps on the roll-rig device in the DNW-TWG Göttingen.

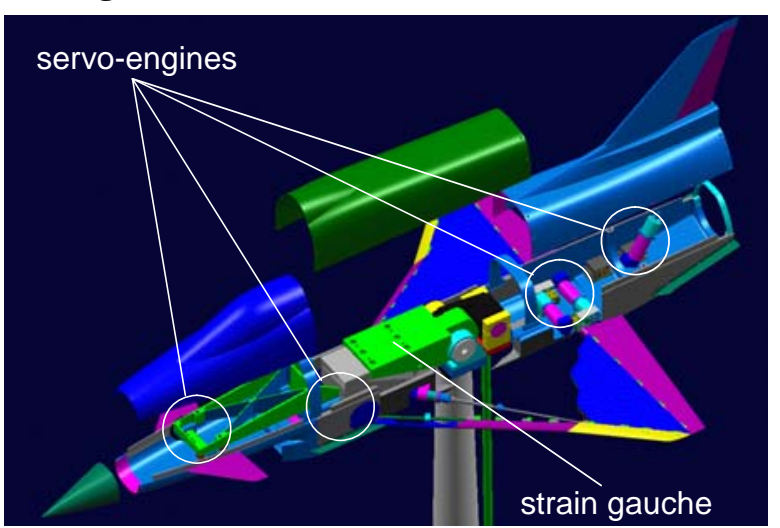

Figure 5: CATIA image of the $\mathrm{X}-31$ wind tunnel model.

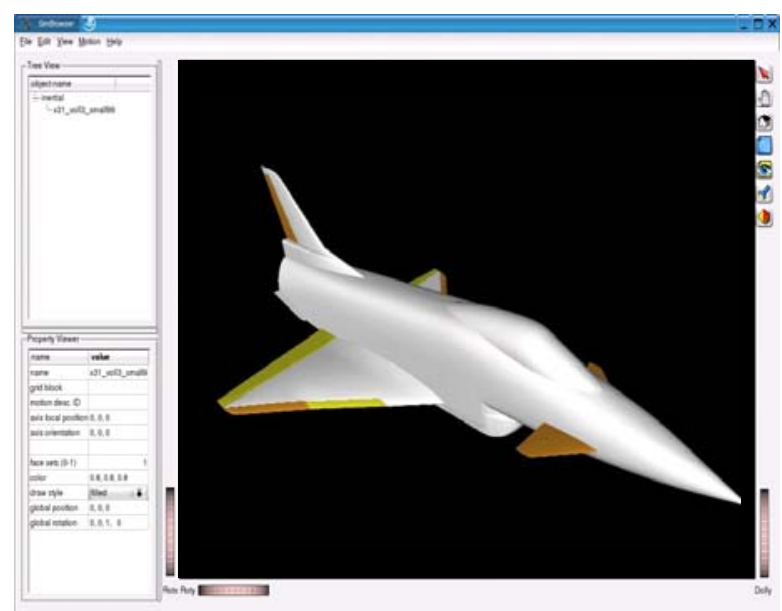

Figure 2: Graphical user interface of the framework environment.

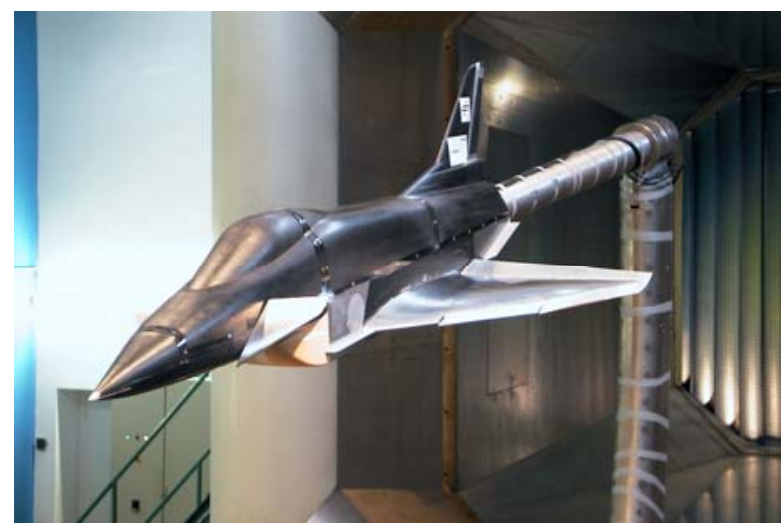

Figure 4: X-31-Remote-Control-Model in the DNWNWB Braunschweig.

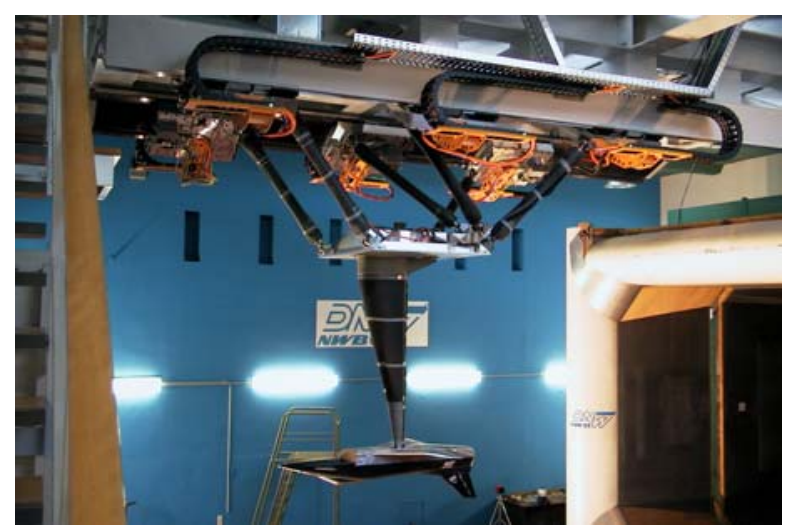

Figure 6: X-31 model on the Model-PositioningMechanism in the DNW-NWB wind tunnel. 


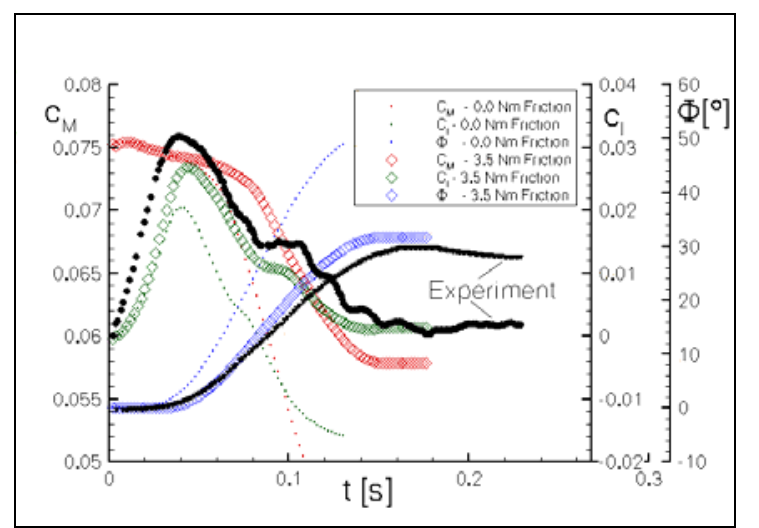

Figure 7: 1 DoF Free-to-Roll maneuver of delta-wingflap-conf. through trailing-edge flap deflection. $M=0.5, \operatorname{Re}=3.8 \mathrm{Mio} ., \Theta=17^{\circ}, \Phi_{0}=0^{\circ}, \eta= \pm 5^{\circ}$.

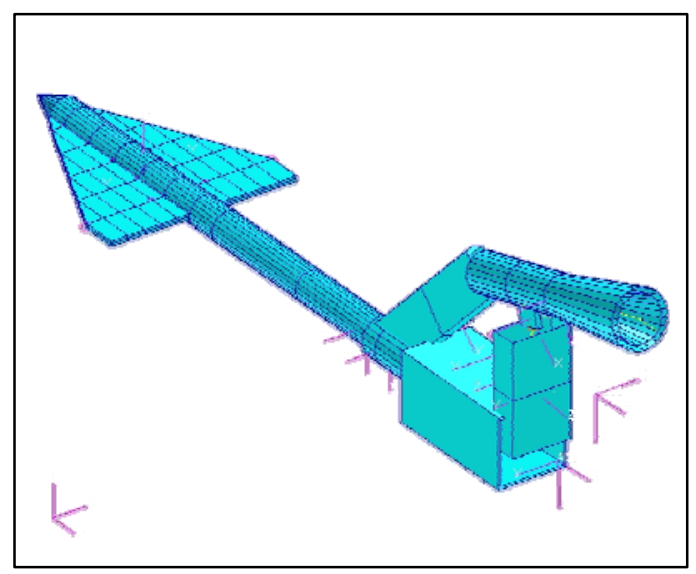

Figure 9: FE-Model of the wind tunnel setup comprising the generic delta-wing and the model support.

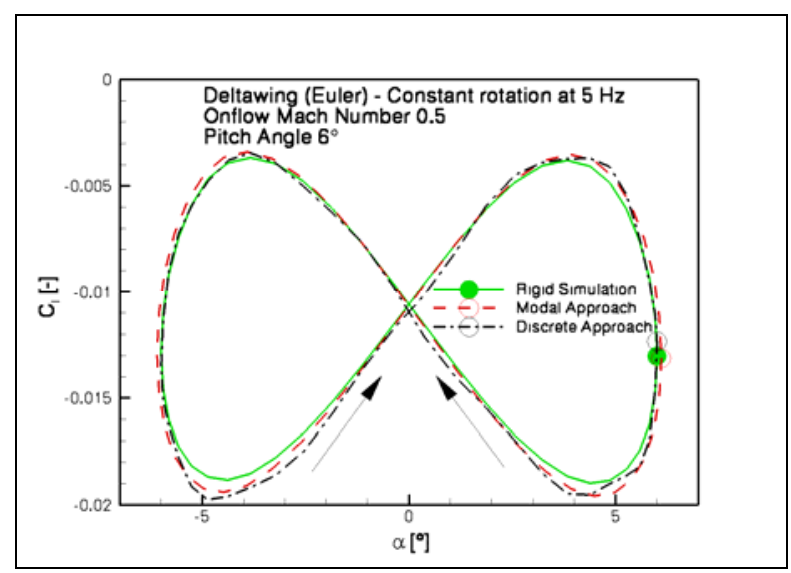

Figure 11: Comparison of rolling moment between rigid- and elastic-body motion of delta-wing during constant rotational movement. Time-accurate coupled CFD(Euler)-CSM simulation.

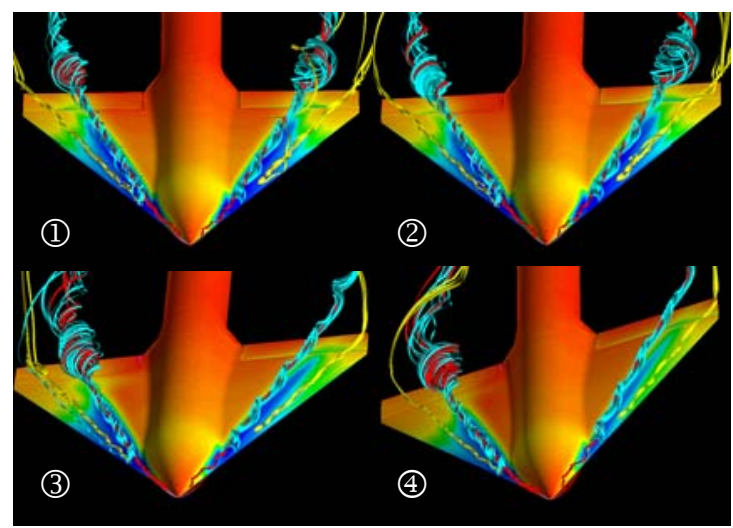

Figure 8: 1 DoF Free-to-Roll maneuver of delta-wingflap-conf. through trailing-edge flap deflection. Flow topology at four different stages. $\mathrm{M}=\mathbf{0 . 5}, \mathrm{Re}=$ 3.8Mio., $\Theta=17^{\circ}, \Phi_{0}=0^{\circ}, \eta= \pm 5^{\circ}$.

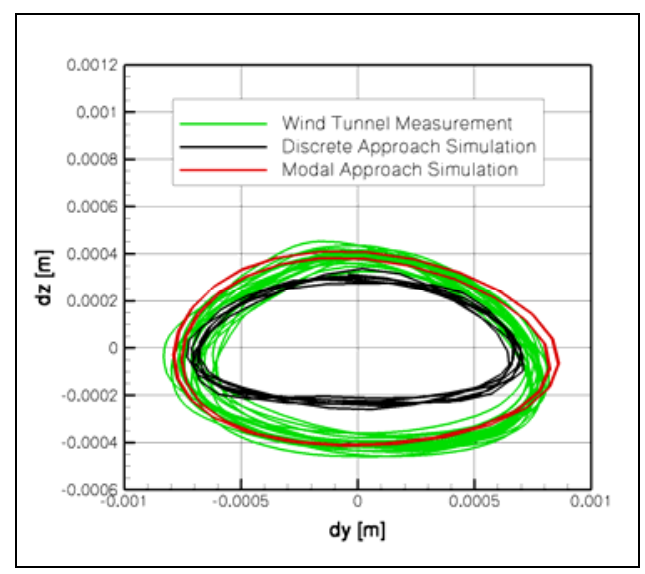

Figure 10: History of the delta-wing sting deflection during elastic-body motion comparison with experiment. Time-accurate coupled CFD(Euler)-CSM simulation.

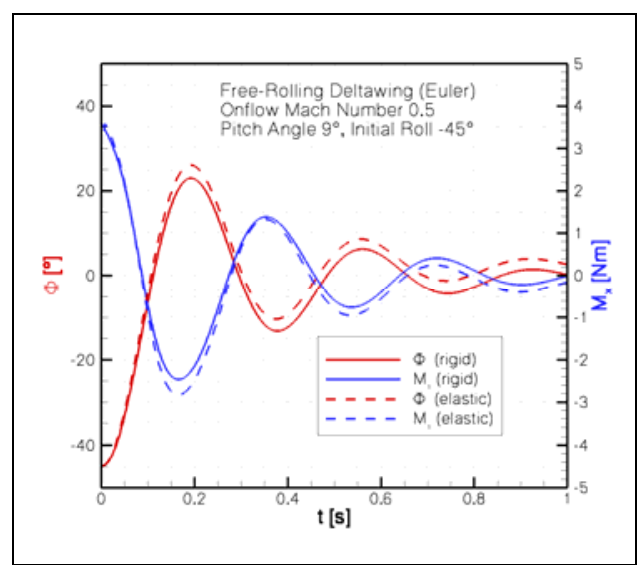

Figure 12: Comparison of rolling moment and rolling angle between rigid- and elastic-body motion of deltawing during free-to-roll motion. Time-accurate coupled CFD(Euler)-CSM-FM simulation. 


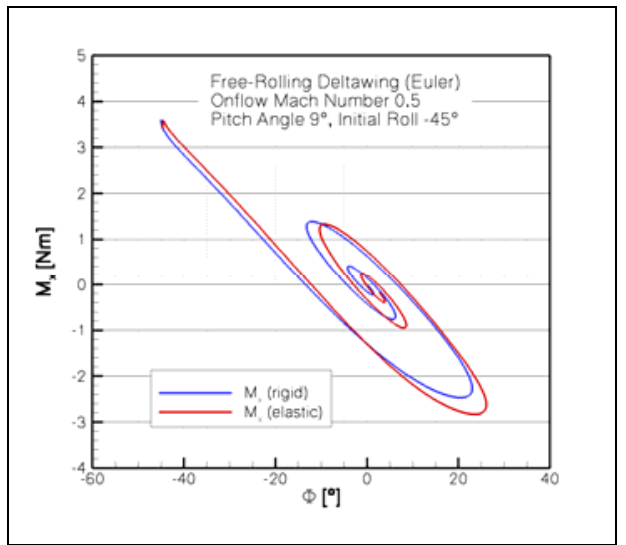

Figure 13: Comparison of rolling moment between rigidand elastic-body motion of delta-wing during free-to-roll motion. Time-accurate coupled CFD(Euler)-CSM-FM.

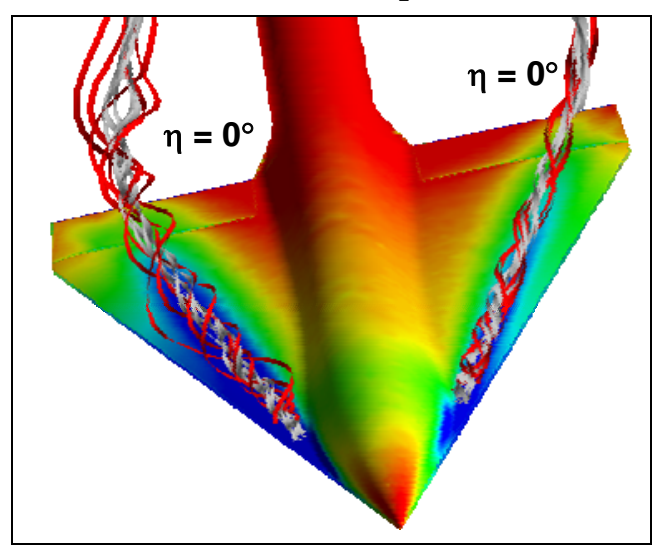

Figure 15: Initial state of trimming scenario. Surface pressure distribution and streamlines. $M=0.5, \Theta=17^{\circ}$, $\Phi=30^{\circ}, \eta_{0}=0^{\circ}$.

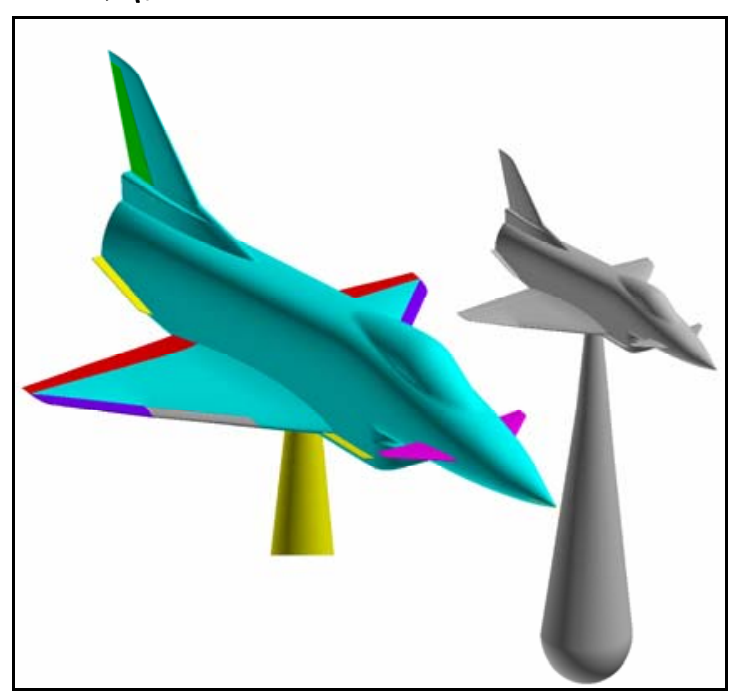

Figure 17: X-31 model topology for the computational simulation.

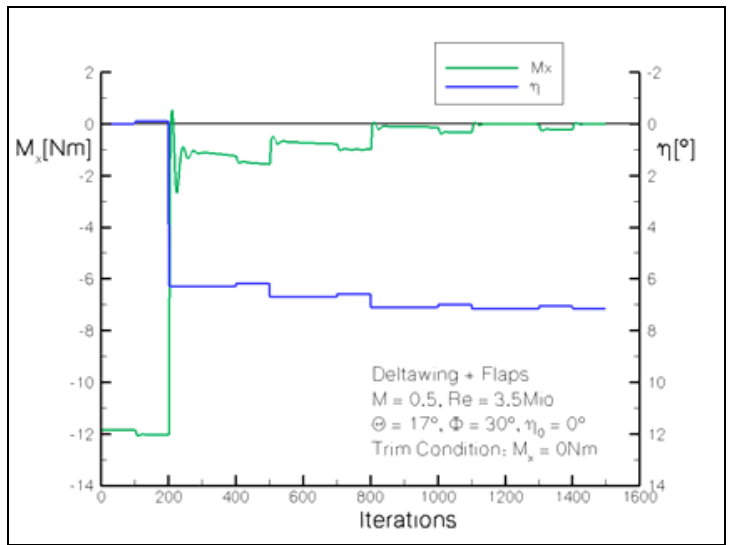

Figure 14: Characteristics of rolling moment $M_{x}$ and flap deflection angle $\eta$ during a trimming scenario.

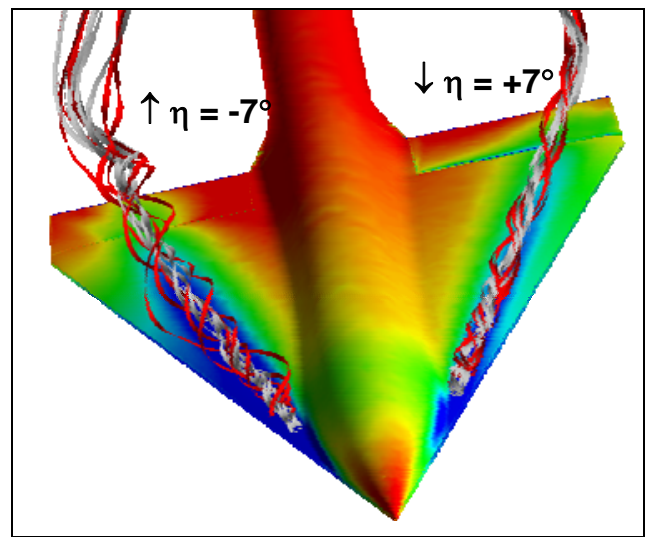

Figure 16: Final state of trimming scenario. Surface pressure distribution and streamlines. $M=0.5, \Theta=17^{\circ}$, $\Phi=30^{\circ}, \eta= \pm 7^{\circ}$.

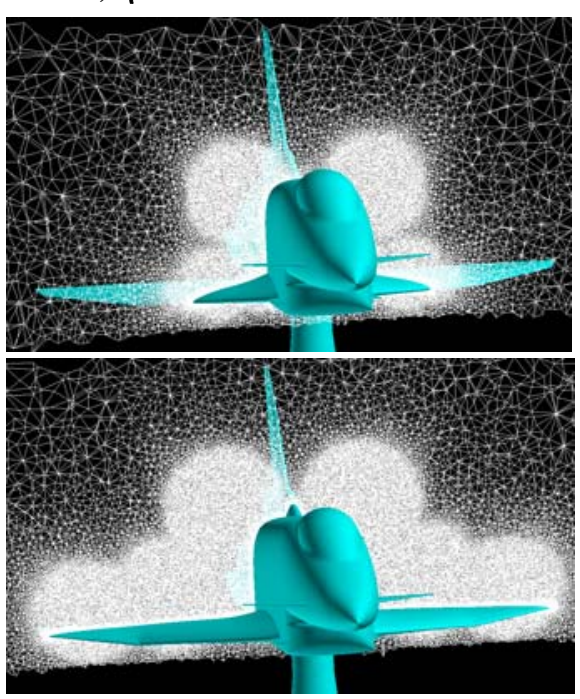

Figure 18: Slices through pre-refined unstructured hybrid mesh of the $\mathrm{X}-31$ configuration. 


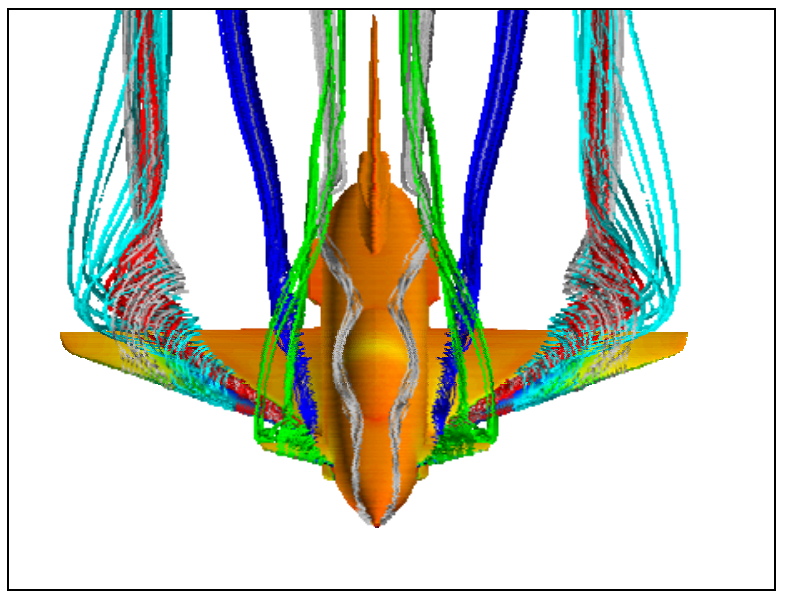

Figure 19: 3D flow field over the $X-31$ configuration at $18^{\circ}$ angle-of-attack.

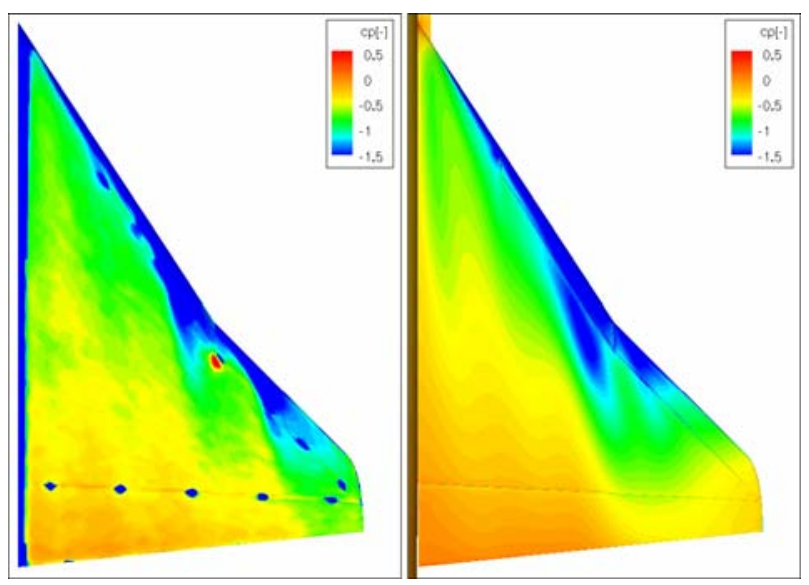

Figure 21: a) Steady PSP measurement of the pressure distribution over the $X-31$ wing. b) Steady TAU RANS calculation of the pressure distribution over the $\mathrm{X}-31$ wing.

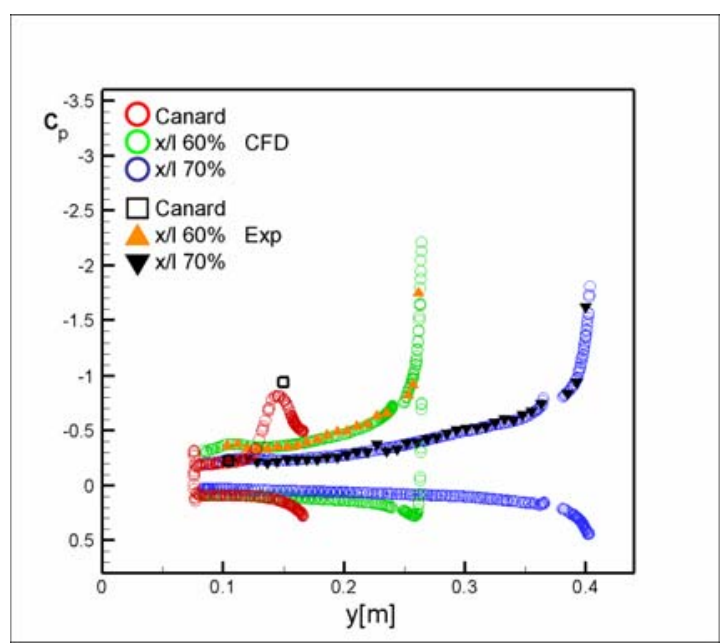

Figure 23: Comparison of the pressure distribution at an angle of attack of $\alpha=10^{\circ}$.

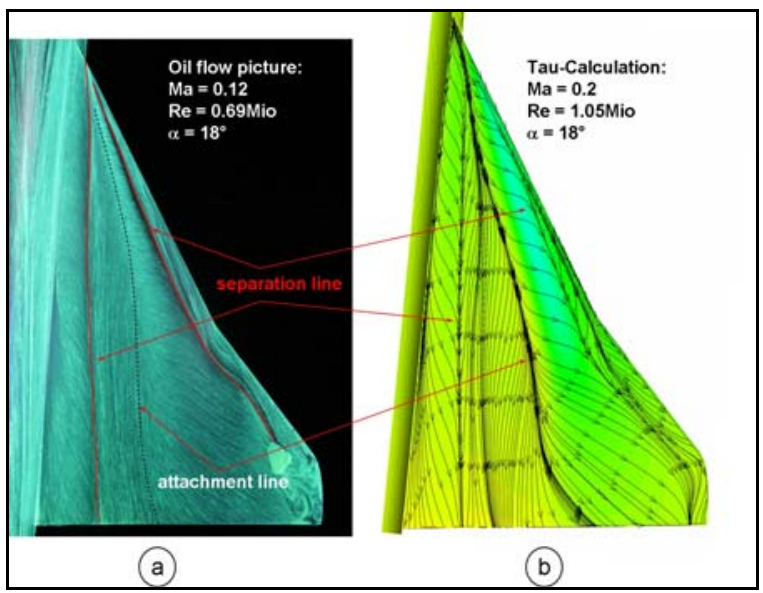

Figure 20: a) Oil flow visualization of the $X-31$ clean wing at $\alpha=18^{\circ}$. b) TAU calculation: Visualization of surface streamlines at $\alpha=18^{\circ}$.

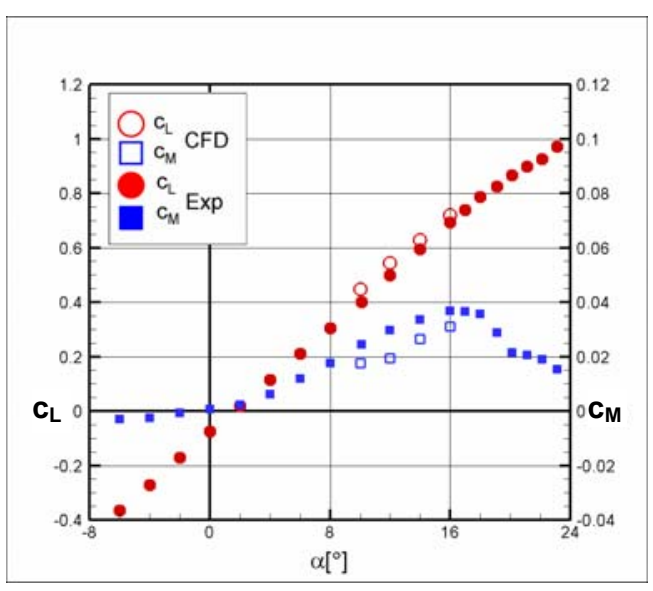

Figure 22: Lift and pitching moment coefficient over angle of attack. Comparison of TAU calculations with experimental data.

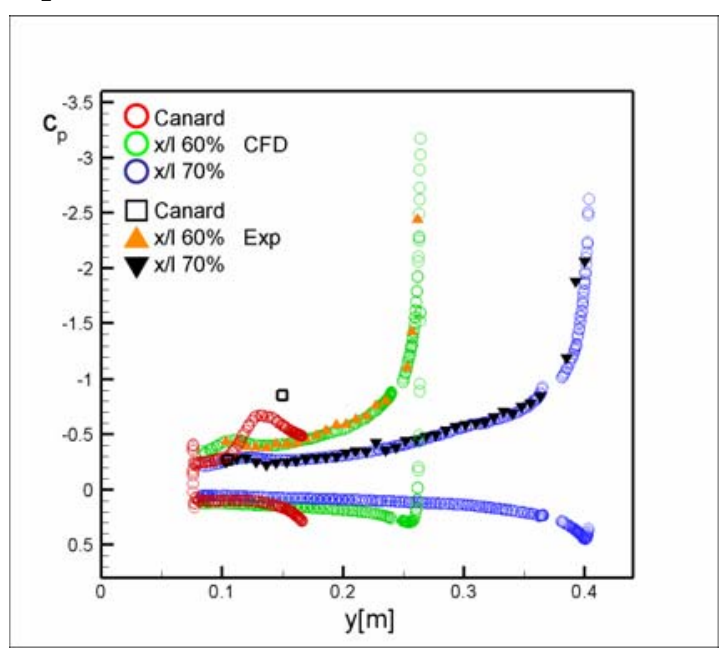

Figure 24: Comparison of the pressure distribution at an angle of attack of $\alpha=12^{\circ}$. 


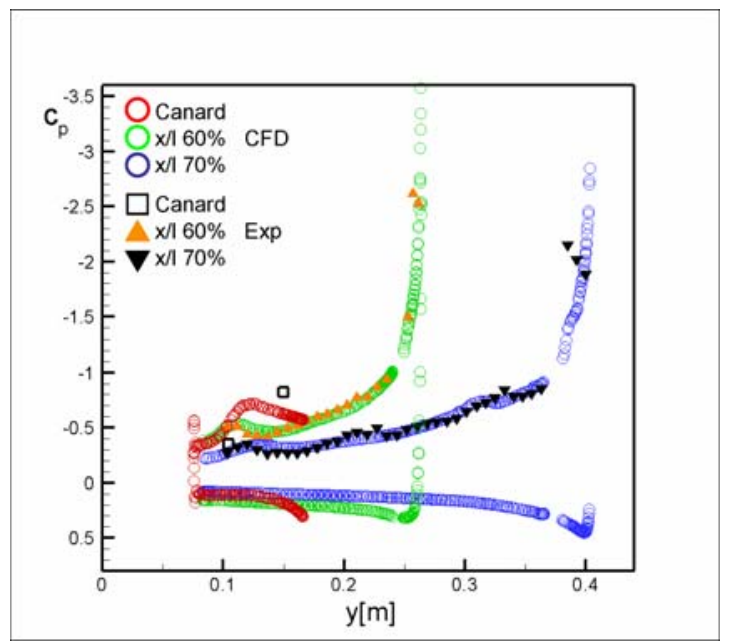

Figure 25: Comparison of the pressure distribution at an angle of attack of $\alpha=12^{\circ}$.

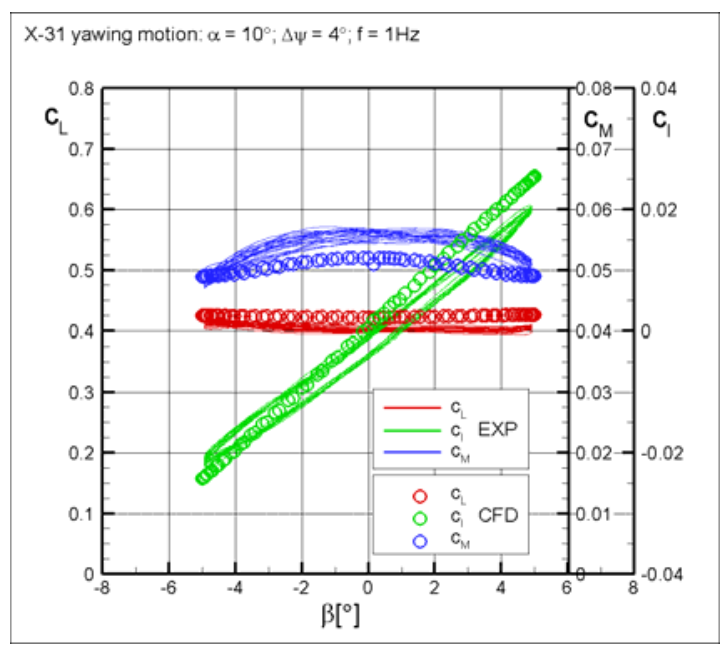

Figure 27: Guided yawing motion of the $X-31$ configuration. Comparison of TAU calculations with experimental data.

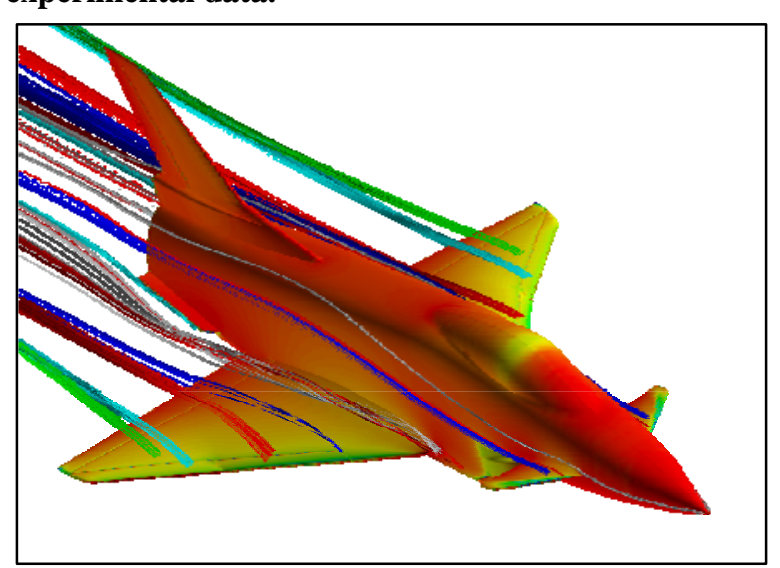

Figure 29: Flow topology during pitching maneuver at $\alpha=6^{\circ}$. Streamlines and surface pressure distribution.

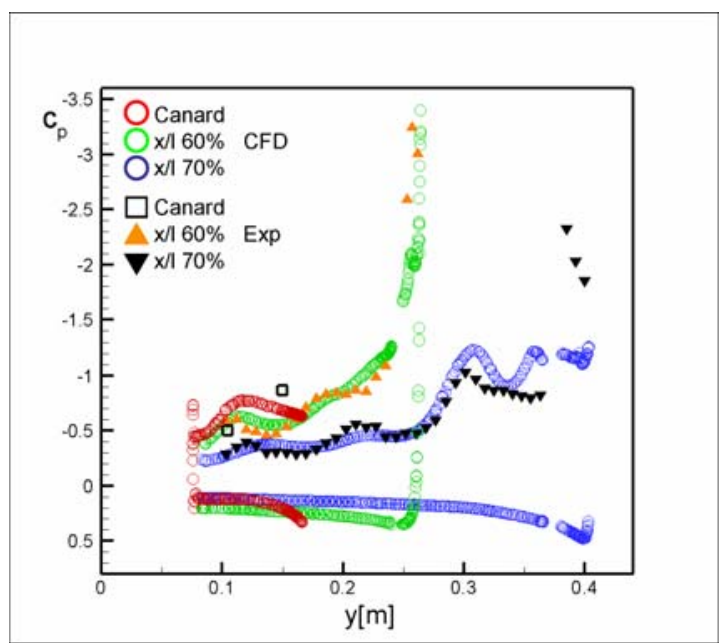

Figure 26: Comparison of the pressure distribution at an angle of attack of $\alpha=10^{\circ}$.

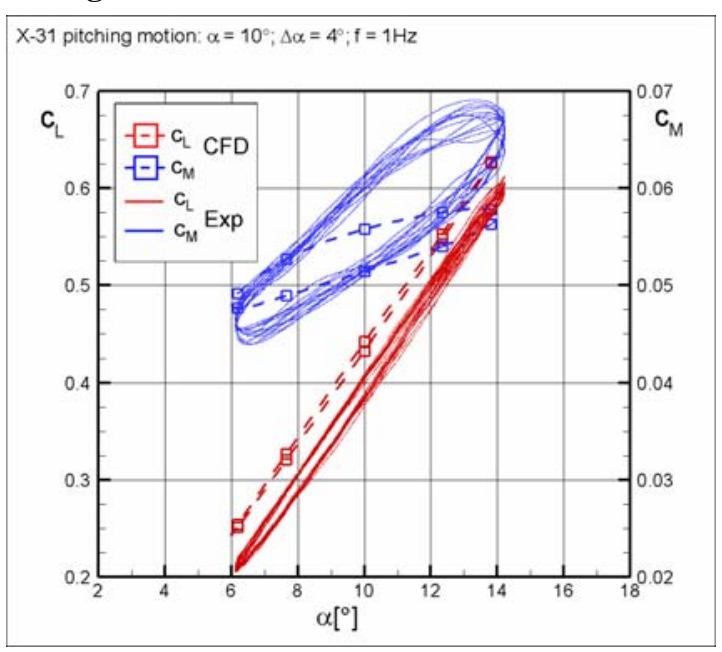

Figure 28: Guided pitching motion of the $X-31$ configuration. Comparison of TAU calculations with experimental data.

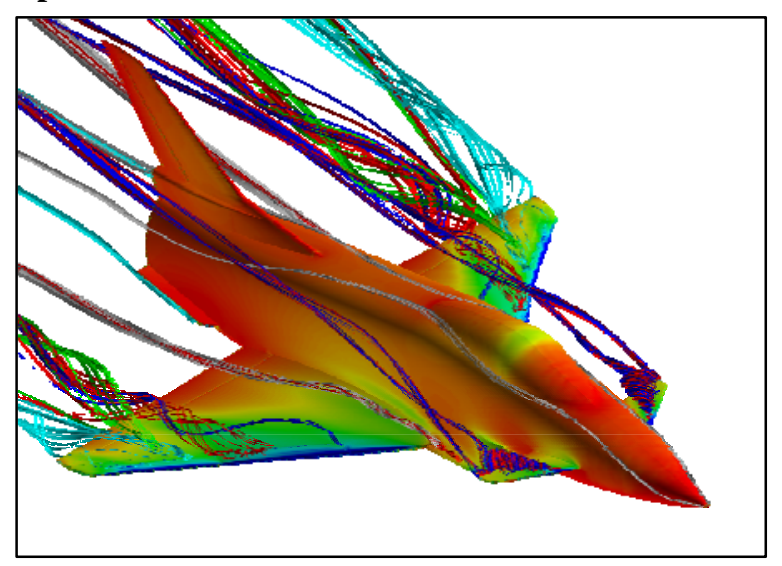

Figure 30: Flow topology during pitching maneuver at $\alpha=14^{\circ}$. Streamlines and surface pressure distribution. 


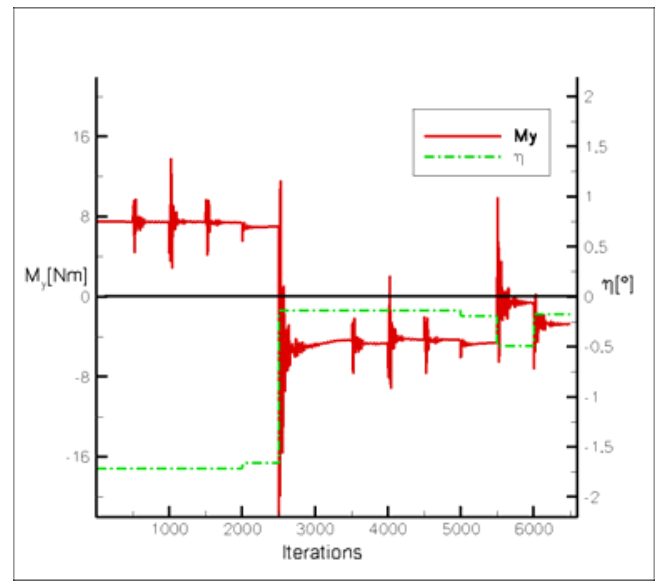

Figure 31: Convergence of the pitching moment during trimming simulation, and the corresponding trailing-edge flap deflection.

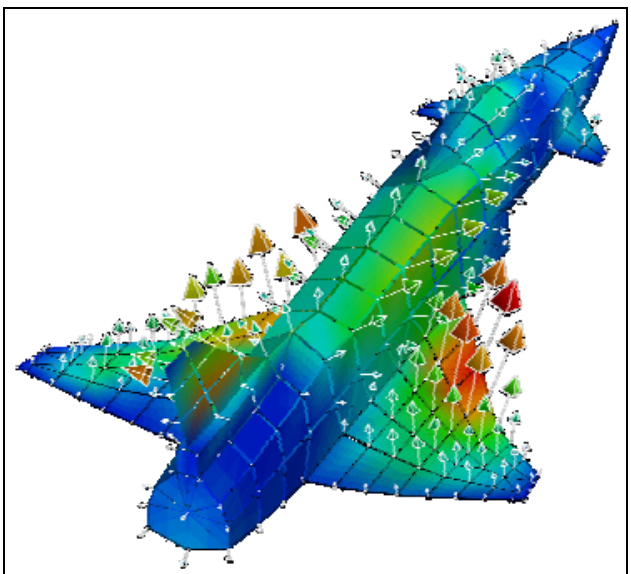

Figure 33: Structural deformation of the FE-Model and corresponding forces on the aircraft. One time step within an unsteady maneuver simulation.

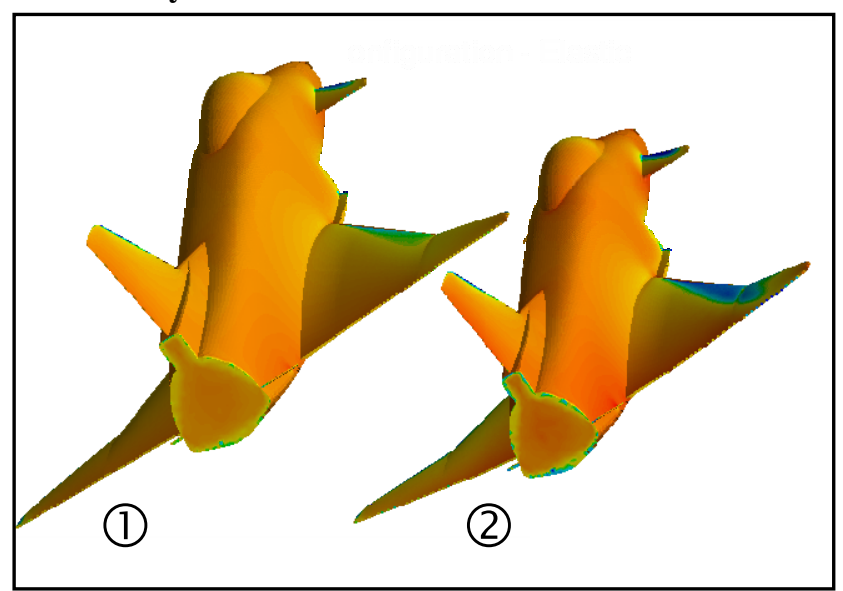

Figure 35: Pressure distribution for the rigid (1) and the elastic (2)CFD-model of the $\mathrm{X}-31$ configuration. Rolling angle at approximately $\Phi=-20^{\circ}$ (at $t=0.2 \mathrm{~s}$ in Fig. 34).

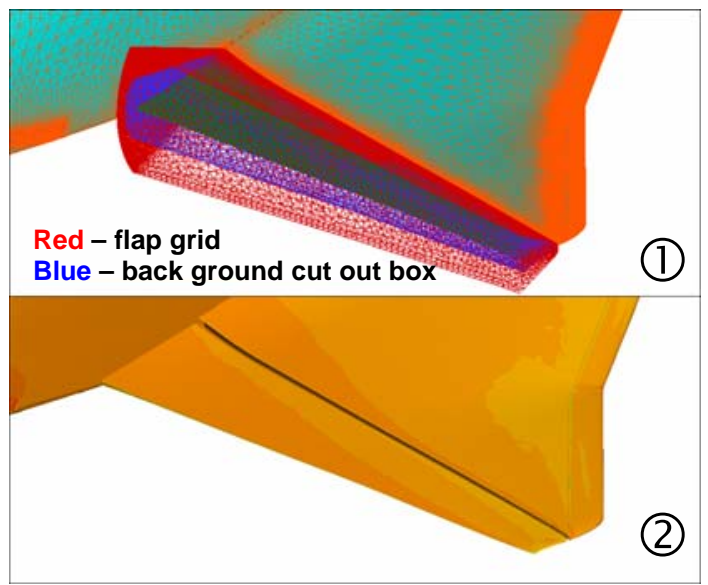

Figure 32: Overlapping chimera-grids at the right trailing-edge flap (1), and the pressure coefficient distribution at the trimmed flight conditions (2).

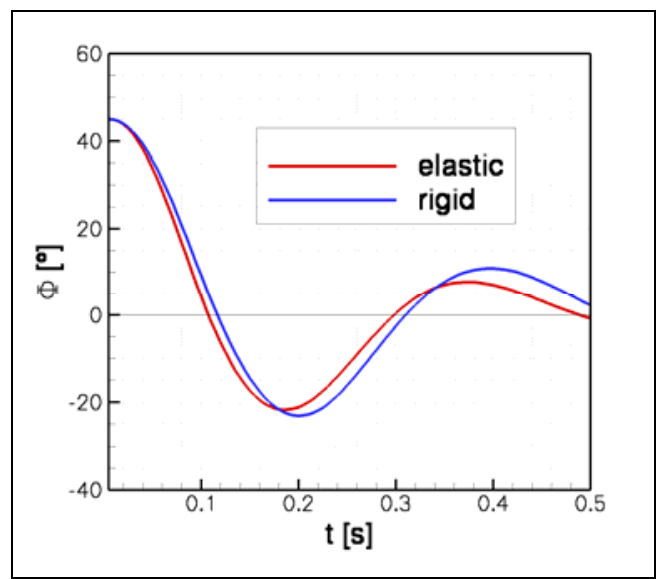

Figure 34: Corresponding rigid and elastic position of the $X-31$ configuration during a free-to-roll maneuver around the longitudinal axis.

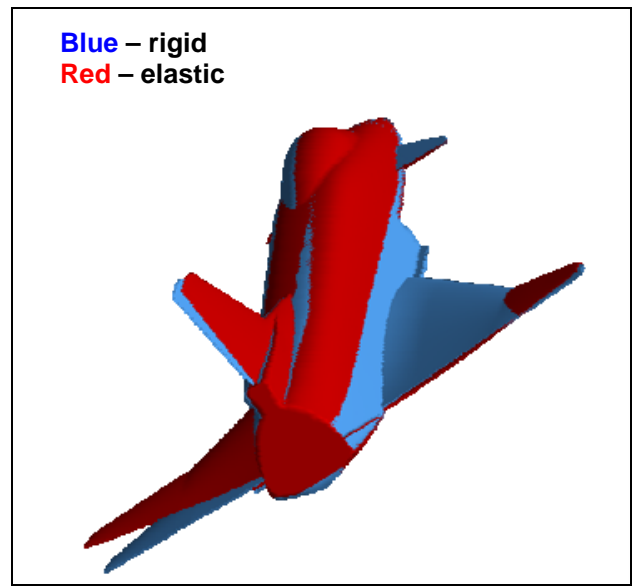

Figure 36: Deformation of elastic CFD-Surface compared to rigid CFD-Surface during the free-to-roll maneuver. 